\title{
On the unitary Cayley graph of a finite ring
}

\author{
Reza Akhtar \\ Department of Mathematics \\ Miami University \\ reza@calico.mth.muohio.edu \\ Tiffany Jackson-Henderson \\ St. Augustine's College \\ tjackhend@yahoo.com \\ Rachel Karpman \\ Scripps College \\ rkarpman@scrippscollege.edu
}

\author{
Megan Boggess \\ Columbia Union College \\ meganboggess@gmail.com \\ Isidora Jiménez \\ Mills College \\ ijimenez@mills.edu \\ Amanda Kinzel \\ Department of Mathematics \\ Purdue University \\ asphilli@math.purdue.edu
}

\author{
Dan Pritikin \\ Department of Mathematics \\ Miami University \\ pritikd@muohio.edu
}

Submitted: May 16, 2009; Accepted: Sep 7, 2009; Published: Sep 18, 2009

Mathematics Subject Classification: 05C25, 05C30

\begin{abstract}
We study the unitary Cayley graph associated to an arbitrary finite ring, determining precisely its diameter, girth, eigenvalues, vertex and edge connectivity, and vertex and edge chromatic number. We also compute its automorphism group, settling a question of Klotz and Sander. In addition, we classify all planar graphs and perfect graphs within this class.
\end{abstract}

\section{Introduction}

Given an integer $n$, consider the graph $\operatorname{Cay}\left(\mathbb{Z}_{n}, \mathbb{Z}_{n}^{*}\right)$ with vertex set $\mathbb{Z}_{n}$ (the integers modulo $n$ ), with vertices $x$ and $y$ adjacent exactly when $x-y$ is a unit in (the ring) $\mathbb{Z}_{n}$. These so-called unitary Cayley graphs have been studied as objects of independent interest (see, for example, [2], [3], [7], [8], [9]) but are of particular relevance in the study of graph 
representations, begun in [5] and continued in many other papers. A graph is said to be representable modulo $n$ if it is isomorphic to an induced subgraph of $\operatorname{Cay}\left(\mathbb{Z}_{n}, \mathbb{Z}_{n}^{*}\right)$; the central problem in graph representations is to determine the smallest positive $n$ modulo which a given graph $G$ is representable. It is natural, then, to study unitary Cayley graphs in the hope of gaining insight into the graph representation problem.

A generalization of unitary Cayley graphs presents itself readily: given a finite ring $R$ (commutative, with unit element $1 \neq 0$ ), one may define $G_{R}=\operatorname{Cay}\left(R, R^{*}\right)$ to be the ring whose vertex set is $R$, with an edge between $x$ and $y$ if $x-y \in R^{*}$. This construction was introduced in [7] and [8], although it does not appear to have been considered in [9].

This article began as a project to address the question of computing the automorphism group $\operatorname{Aut}\left(\operatorname{Cay}\left(\mathbb{Z}_{n}, \mathbb{Z}_{n}^{*}\right)\right)$, first raised by Klotz and Sander in [9]. We soon realized that it was more natural to consider this question in the context of (unitary Cayley graphs of) finite rings. In this article we give a complete answer to this question; moreover, we extend the results of [9] to the setting of finite rings and explore various other graphtheoretic properties not considered there. Our proofs emphasize the dependence of results on the underlying algebraic structure of the rings concerned; in some cases, these provide a considerable simplification of the Klotz-Sander proofs. We hope that the use of some algebra will provide a more cohesive approach to further study of these graphs.

A key observation is the following: since $R$ is a finite ring, it is Artinian, and hence $R \cong R_{1} \times \ldots \times R_{t}$, where each $R_{i}$ is a finite local ring with maximal ideal $\mathfrak{m}_{i}$. Since $\left(u_{1}, \ldots, u_{t}\right)$ is a unit of $R$ if and only if each $u_{i}$ is a unit in $R_{i}^{*}$, we see immediately that $G_{R}$ is the conjunction (sometimes called tensor product or Kronecker product) of the graphs $G_{R_{1}}, \ldots, G_{R_{t}}$. Moreover, if $x, y \in R_{i},\{x, y\}$ is an edge of $G_{R_{i}}$ if and only if

$x-y \notin \mathfrak{m}_{i}$. It follows immediately that each $G_{R_{i}}$ is a complete balanced multipartite graph whose partite sets are the cosets of $\mathfrak{m}_{i}$ in (the additive group) $R_{i}$. This perspective allows us, for example, to give a simple, explicit computation of the eigenvalues of the graphs $G_{R}$ (see Section 10). In future work we hope to generalize the study of graph representations to this broader setting.

Part of this research was carried out in the SUMSRI program, held at Miami University during the summer of 2008. We thank Miami University, the National Security Agency, and the National Science Foundation for their support of the first six authors. We also thank the referee for suggestions which helped improve this paper.

\section{Algebraic Background and Basic Properties}

Throughout this paper, all rings mentioned are commutative with unit element $1 \neq 0$. Let $R$ be a finite ring. Since $R$ is Artinian, the structure theorem [4, p. 752, Theorem 3] implies that $R \cong R_{1} \times \ldots \times R_{t}$, where each $R_{i}$ is a finite local ring with maximal ideal $\mathfrak{m}_{i}$; this decomposition is unique up to permutation of factors. We denote by $k_{i}$ the (finite) residue field $R_{i} / \mathfrak{m}_{i}, \pi_{i}: R_{i} \rightarrow k_{i}$ the quotient map, and $f_{i}=\left|k_{i}\right|$. We also assume (after appropriate permutation of factors) that $f_{1} \leqslant f_{2} \leqslant \ldots \leqslant f_{t}$. This notation will be 
maintained throughout the paper whenever $R$ is mentioned as a finite (or more generally Artinian) ring.

The following proposition is well-known, but we include it here for the sake of completeness.

Proposition 2.1. Let $S$ be a finite local ring with maximal ideal $\mathfrak{m}$. Then there exists a prime $p$ such that $|R|,|\mathfrak{m}|$ and $|R / \mathfrak{m}|$ are all powers of $p$.

\section{Proof.}

Since $k=R / \mathfrak{m}$ is a field, its order must be equal to $p^{e}$ for some prime $p$ and integer $e \geqslant 1$. Since $R$ is finite, Nakayama's Lemma implies that as long as $\mathfrak{m}^{i} \neq 0, \mathfrak{m m}^{i}=$ $\mathfrak{m}^{i+1} \neq \mathfrak{m}^{i}$; that is, $\mathfrak{m}^{i+1}$ is a strict subset of $\mathfrak{m}^{i}$. Since $R$ is finite, this implies that in the chain $R \supseteq \mathfrak{m} \supseteq \mathfrak{m}^{2} \supseteq \ldots$, there is some $i$ such that $\mathfrak{m}^{i}=0$. Then for all $j \geqslant 1,\left|\mathfrak{m}^{j-1} / \mathfrak{m}^{j}\right|$ is a $k$-vector space, so its order is a power of $p$. Then descending induction on $j$ shows that $\left|\mathfrak{m}^{j}\right|$ is a power of $p$ for all $j \geqslant 0$.

We note in particular that the nilradical of a local ring $R$ (the ideal $\mathfrak{N}_{R}$ of nilpotent elements) is simply the (unique) maximal ideal of $R$.

It is well-known that if $R$ is an Artinian ring, then $R \cong R_{1} \times \ldots \times R_{t}$, where each $R_{i}$ is an Artinian local ring. Furthermore, $R^{*}=R_{1}^{*} \times \ldots \times R_{t}^{*}$, and hence two vertices $x=\left(x_{1}, \ldots, x_{t}\right), y=\left(y_{1}, \ldots, y_{t}\right)$ are adjacent if and only if $x_{i}-y_{i} \in R_{i}^{*}$ for all $i=1, \ldots, t$. Equivalently, $x$ is adjacent to $y$ if and only if for each $i=1, \ldots, t, x_{i}-y_{i} \notin \mathfrak{m}_{i}$; that is, $\pi_{i}\left(x_{i}\right) \neq \pi_{i}\left(y_{i}\right)$.

The following are some basic consequences of this definition:

\section{Proposition 2.2.}

- Let $R$ be any ring. Then $G_{R}$ is a regular graph of degree $\left|R^{*}\right|$.

- Let $S$ be a local ring with maximal ideal $\mathfrak{m}$. Then $G_{S}$ is a complete multipartite graph whose partite sets are the cosets of $\mathfrak{m}$ in $S$. In particular, $G_{S}$ is a complete graph if and only if $S$ is a field.

- If $R$ is any Artinian ring and $R \cong R_{1} \times \ldots \times R_{t}$ as a product of local rings, then $G_{R}=\bigwedge_{i=1}^{t} G_{R_{i}}$. Hence, $G_{R}$ is a conjunction of complete multipartite graphs.

\section{Proof.}

The first statement follows from the fact that the neighborhood of any vertex $a$ is $\left\{a+u: u \in R^{*}\right\}$. For the second statement, simply note that $x, y \in S$ are adjacent if and only if $x-y \notin \mathfrak{m}$ and that $S$ is a field if and only if $\mathfrak{m}=0$. The third statement follows from the fact that $R^{*} \cong R_{1}^{*} \times \ldots \times R_{t}^{*}$. 


\section{Remark.}

For any $r \in R$, the map $z \mapsto z+r$ defines an automorphism $G_{R}$; similarly, if $u \in R^{*}$, $z \mapsto u z$ is also an automorphism of $G_{R}$. We will compute the full group $\operatorname{Aut}\left(G_{R}\right)$ in Section 4.

Throughout this paper, we use $N(v)$ for the neighborhood of a vertex (that is, the set of vertices adjacent to $v)$ and $N(u, v)$ for the number of common neighbors of the vertices $u$ and $v$. We now give a formula for the latter in $G_{R}$ :

Proposition 2.3. Suppose $a=\left(a_{1}, \ldots, a_{t}\right)$ and $b=\left(b_{1}, \ldots, b_{t}\right)$ are vertices of $G_{R}$. Let $I=\left\{i: 1 \leqslant i \leqslant t, \pi_{i}\left(a_{i}\right)=\pi_{i}\left(b_{i}\right)\right\}$ and $J=\{1, \ldots, t\}-I$. Then

$$
N(a, b)=|R| \prod_{i \in I}\left(1-\frac{1}{f_{i}}\right) \prod_{j \in J}\left(1-\frac{2}{f_{j}}\right)
$$

\section{Proof.}

If $c=\left(c_{1}, \ldots, c_{t}\right)$ is adjacent to both $a$ and $b$, then for each $k=1, \ldots, t, c_{i}$ may be any element such that $\pi_{i}\left(c_{i}\right) \notin\left\{\pi_{i}\left(a_{i}\right), \pi_{i}\left(b_{i}\right)\right\}$. If $\pi_{i}\left(a_{i}\right)=\pi_{i}\left(b_{i}\right)$, there are $\frac{f_{i}-1}{f_{i}}\left|R_{i}\right|$ choices for $c_{i}$, and if $\pi_{i}\left(a_{i}\right) \neq \pi_{i}\left(b_{i}\right)$, there are $\frac{f_{i}-2}{f_{i}}\left|R_{i}\right|$ choices for $c_{i}$. In total, then, there are $\prod_{i \in I}\left(1-\frac{1}{f_{i}}\right)\left|R_{i}\right| \cdot \prod_{j \in J}\left(1-\frac{2}{f_{j}}\right)\left|R_{j}\right|=|R| \prod_{i \in I}\left(1-\frac{1}{f_{i}}\right) \prod_{j \in J}\left(1-\frac{2}{f_{j}}\right)$ choices for $c$.

Corollary 2.4. Let $R$ be an Artinian ring and $x, y \in G_{R}$. Then $N(x)=N(y)$ if and only if $x-y \in \mathfrak{N}_{R}$.

Some questions about properties of unitary Cayley graphs are best viewed as purely combinatorial questions about conjunctions of complete balanced multipartite graphs. We will adopt this persective at various points later in this article. Sometimes we can simplify things even further, as explained in the next paragraph.

Consider two vertices $v, w$ of a graph $G$ to be equivalent when $N(v)=N(w)$. Then, following [6] we define the reduction of $G$ to be the graph $G_{r e d}$ whose vertex set is the set of equivalence classes of vertices (as defined above), and whose edges consist of pairs $\{A, B\}$ of equivalence classes with the property that $A \cup B$ induces a complete bipartite subgraph of $G$.

Proposition 2.5. Let $R$ be an Artinian ring. Then the reduction $\left(G_{R}\right)_{\text {red }} \cong G_{R_{\text {red }}}$ where $R_{\text {red }}=R / \mathfrak{N}_{R}$ is the (ring-theoretic) reduction of $R$.

\section{Proof.}

First, write $R=R_{1} \times \ldots \times R_{t}$ as a product of local rings. Then $\mathfrak{N}_{R}=\mathfrak{N}_{R_{1}} \times \ldots \times \mathfrak{N}_{R_{t}}=$ $\mathfrak{m}_{1} \times \ldots \times \mathfrak{m}_{t}$ and so $R_{\text {red }}=R_{1} / \mathfrak{m}_{1} \times \ldots \times R_{t} / \mathfrak{m}_{t}$ is a product of fields. Moreover, it is clear from the description of adjacency above that two vertices $\left(a_{1}, \ldots, a_{t}\right),\left(b_{1}, \ldots, b_{t}\right)$ of $G_{R}$ have the same neighborhood if and only if $a_{i}-b_{i} \in \mathfrak{m}_{i}$ for all $i=1, \ldots, t$. This implies 
that vertices of $\left(G_{R}\right)_{\text {red }}$ correspond to elements of $R_{\text {red }}=R_{1} / \mathfrak{m}_{1} \times \ldots \times R_{t} / \mathfrak{m}_{t}$. Since adjacency is defined by the same rule in both graphs, it follows that $\left(G_{R}\right)_{r e d} \cong G_{R_{\text {red }}}$.

Proposition 2.5 allows us to convert general questions about unitary Cayley graphs of finite rings to corresponding questions about finite reduced rings (i.e. products of fields).

\section{Diameter and Girth}

In the following we use $\operatorname{diam}(G)$ and $\operatorname{gr}(G)$ (respectively) to denote the diameter and girth of a graph $G$.

Theorem 3.1. Let $R=R_{1} \times \ldots \times R_{t}$ be an Artinian ring. Then

$$
\operatorname{diam} G_{R}= \begin{cases}1 & \text { if } t=1 \text { and } R \text { is a field } \\ 2 & \text { if } t=1 \text { and } R \text { is not a field } \\ 2 & \text { if } t \geqslant 2, f_{1} \geqslant 3 \\ 3 & \text { if } t \geqslant 2, f_{1}=2, f_{2} \geqslant 3 \\ \infty & \text { if } t \geqslant 2, f_{1}=f_{2}=2 .\end{cases}
$$

\section{Proof.}

If $t=1$, then by Proposition $2.2, G_{R}$ is complete if $R$ is a field, and is a complete multipartite graph (with at least two partite sets) if $R$ is not a field. In the first case, $G_{R}$ has diameter 1 ; in the second case, it has diameter 2 . Now suppose $t \geqslant 2$ and $f_{1}>2$. Then $f_{i} \geqslant 3$ for all $i=1, \ldots, t$, so given distinct vertices $a=\left(a_{1}, \ldots, a_{t}\right), b=\left(b_{1}, \ldots, b_{t}\right)$, select elements $c_{i} \in R_{i}, i=1, \ldots, t$, such that $\pi_{i}\left(c_{i}\right) \notin\left\{\pi_{i}\left(a_{i}\right), \pi_{i}\left(b_{i}\right)\right\}$. Then $c=\left(c_{1}, \ldots, c_{t}\right)$ is a common neighbor of $a$ and $b$ and so diam $G_{R} \leqslant 2$. Obviously $G_{R}$ is not complete in this case, so diam $G_{R}=2$.

If $t \geqslant 2$ and $f_{1}=2$, observe that the vertices $(0,0, \ldots, 0)$ and $(1,0, \ldots, 0)$ are neither adjacent nor do they share a common neighbor; hence diam $G_{R} \geqslant 3$. If, moreover, $f_{2}=2$, then there is a no path in $G_{R}$ between these same two vertices, so $G_{R}$ is disconnected. On the other hand, if $f_{2} \geqslant 3$, consider distinct vertices $a=\left(a_{1}, \ldots, a_{t}\right)$ and $b=\left(b_{1}, \ldots, b_{t}\right)$ such that $d(a, b) \geqslant 3$. In particular, $\pi_{1}\left(a_{1}\right) \neq \pi_{1}\left(b_{1}\right)$ and for some $i \geqslant 2, \pi_{i}\left(a_{i}\right)=$ $\pi_{i}\left(b_{i}\right)$. Now define $c=\left(c_{1}, \ldots, c_{t}\right), d=\left(d_{1}, \ldots, d_{t}\right)$ as follows: for each $i, 1 \leqslant i \leqslant t$, if $\pi_{i}\left(a_{i}\right)=\pi_{i}\left(b_{i}\right)$, pick $c_{i}, d_{i} \in R_{i}$ such that $\pi_{i}\left(c_{i}\right), \pi_{i}\left(d_{i}\right)$, and $\pi_{i}\left(a_{i}\right)=\pi_{i}\left(b_{i}\right)$ are distinct; if $\pi_{i}\left(a_{i}\right) \neq \pi_{i}\left(b_{i}\right)$, set $d_{i}=b_{i}$ and $c_{i}=a_{i}$. Then $a, d, c, b$ is a path of length 3 , so $\operatorname{diam} G_{R}=3$.

Theorem 3.2. $g r G_{R}= \begin{cases}3 & \text { if } f_{1} \geqslant 3 \\ 6 & \text { if } R \cong \mathbb{Z}_{2}^{r} \times \mathbb{Z}_{3} \text { for some } r \geqslant 1 \\ \infty & \text { if } R \cong \mathbb{Z}_{2}^{r} \text { for some } r \geqslant 1 \\ 4 & \text { otherwise. }\end{cases}$

\section{Proof.}

Suppose first that $f_{1} \geqslant 3$. Then any three vertices $a=\left(a_{1}, \ldots, a_{t}\right), b=\left(b_{1}, \ldots, b_{t}\right)$, $c=\left(c_{1}, \ldots, c_{t}\right)$ such that $\pi_{i}\left(a_{i}\right), \pi_{i}\left(b_{i}\right)$, and $\pi_{i}\left(c_{i}\right)$ are distinct for all $i=1, \ldots, t$ induce a triangle, and so gr $G_{R}=3$. 
We next consider the case $t=1, f_{1}=2$. If $R \cong \mathbb{Z}_{2}$, clearly gr $G_{R}=\infty$. Otherwise, $R$ is not a field, so $G_{R}$ is a complete bipartite graph with partite sets of size $\left|\mathfrak{m}_{1}\right| \geqslant 2$, and hence gr $G_{R}=4$.

Now suppose $f_{1}=2$ and $t \geqslant 2$. Then $G_{R}$ is a bipartite graph, so gr $\left(G_{R}\right) \geqslant 4$. Let $a=(0, \ldots, 0)$ and $b=(1, \ldots, 1)$. If $R_{i}$ is not a field for some $i \geqslant 1$, then $\left|\mathfrak{m}_{i}\right| \geqslant 2$, so choosing $0 \neq x \in \mathfrak{m}_{i}$, define $c=\left(c_{1}, \ldots, c_{t}\right)$ and $d=\left(d_{1}, \ldots, d_{t}\right)$ by setting, for each $j=1, \ldots, t, c_{j}=\delta_{i j} x$ and $d_{j}=1+\delta_{i j} x$. Then $a, b, c, d, a$ is a 4-cycle, and so gr $\left(G_{R}\right)=4$. If $R_{j}$ is a field for all $j \geqslant 1$ and $\left|R_{i}\right| \geqslant 4$ for some $i$, choose elements $c_{i}, d_{i} \in R_{i}$ such that $\pi_{i}\left(c_{i}\right), \pi_{i}\left(d_{i}\right)$ are distinct elements of $k-\{0,1\}$. For $j \neq i$, define $c_{j}=a_{j}$ and $d_{j}=b_{j}$, and let $c=\left(c_{1}, \ldots, c_{t}\right), d=\left(d_{1}, \ldots, d_{t}\right)$. Then $a, b, c, d, a$ is a 4-cycle, and so gr $\left(G_{R}\right)=4$ in this case, too.

We are now reduced to the case that $R \cong \mathbb{Z}_{2}^{r} \times \mathbb{Z}_{3}^{s}$ for some $r, s, r+s \geqslant 2$. Since $G_{R}$ is bipartite, it contains no odd cycles. To simplify notation in the following discussion we use the notation $x_{m}$ to represent an $m$-tuple each of whose coordinates is $x$ (in the appropriate ring). If $s \geqslant 2$, then $\left(0_{r}, 0_{s}\right),\left(1_{r}, 1_{s}\right),\left(0_{r}, 2, \ldots, 2,0\right),\left(1_{r}, 1, \ldots, 1,2\right),\left(0_{r}, 0_{s}\right)$ defines a 4-cycle in $G_{R}$. If $s=1$, the vertex sequence $\left(0_{r}, 0\right),\left(1_{r}, 1\right),\left(0_{r}, 2\right),\left(1_{r}, 0\right)$ $\left(0_{r}, 1\right),\left(1_{r}, 2\right),\left(0_{r}, 0\right)$ defines a 6 -cycle, so gr $G_{R} \leqslant 6$. If $a, b, c, d, a$ were a cycle of length 4 in $G_{R}$, then $a_{i}=c_{i}$ for all $i$ and $b_{i}=d_{i}(1 \leqslant i \leqslant r)$, so in particular $a_{r+1} \neq c_{r+1}$, $b_{r+1} \neq d_{r+1}$, and so $S=\left\{a_{r+1}, c_{r+1}\right\}$ and $T=\left\{b_{d+1}, d_{r+1}\right\}$ are (by virtue of the adjacency conditions) disjoint subsets of $R_{r+1}$, each of cardinality 2 . However, $\left|R_{r+1}\right|=3$, so this is a contradiction. Thus, gr $G_{R}=6$. The last case to consider is $R \cong \mathbb{Z}_{2}^{r}$, but in this case, $G_{R} \cong 2^{r-1} K_{2}$ and hence gr $G_{R}=\infty$.

Corollary 3.3. The number of triangles in $G_{R}$ is $\frac{|R|^{3}}{6} \prod_{i=1}^{t}\left(1-\frac{1}{f_{i}}\right)\left(1-\frac{2}{f_{i}}\right)$.

\section{Proof.}

If $f_{1}=2$, then by Proposition $3.2, G_{R}$ is triangle-free, so the claim holds in this case. If $f_{1} \geqslant 3$, then given a vertex $a \in R$, by Proposition 2.2 there are $\left|R^{*}\right|=|R| \prod_{i=1}^{t}\left(1-\frac{1}{f_{i}}\right)$ choices for an adjacent vertex $b$. Now, Proposition 2.3 implies that there are $|R| \prod_{i=1}^{t}\left(1-\frac{2}{f_{i}}\right)$ choices for a third vertex which is a common neighbor of both $a$ and $b$. Since any such triangle may be formed in 6 distinct ways, the total number of triangles is $\frac{|R|^{3}}{6} \prod_{i=1}^{t}(1-$ $\left.\frac{1}{f_{i}}\right)\left(1-\frac{2}{f_{i}}\right)$

\section{Automorphisms}

In this section we compute the group $\operatorname{Aut}\left(G_{R}\right)$ when $R$ is a finite ring. We begin by reducing the problem to the case of reduced rings. 
Lemma 4.1. Let $R$ be a finite ring and $n=\left|\mathfrak{N}_{R}\right|$.

Then there is an isomorphism $f:$ Aut $\left(G_{R}\right) \stackrel{\cong}{\rightrightarrows}$ Aut $\left(G_{R_{\text {red }}}\right) \times\left(S_{n}\right)^{\left|R / \mathfrak{N}_{R}\right|}$.

\section{Proof.}

It follows from Corollary 2.4 that any $\sigma \in \operatorname{Aut}\left(G_{R}\right)$ permutes the cosets of $\mathfrak{N}_{R}$ in $R$; in particular, $\sigma$ induces an automorphism $\bar{\sigma} \in A u t\left(G_{R_{\text {red }}}\right)$. Moreover, if one fixes an enumeration $x_{1}, \ldots, x_{n}$ of the elements of $\mathfrak{N}_{R}$ and a set of coset representatives $\mathcal{R}=$ $\left\{a_{C}\right\}_{C \in R / \mathfrak{N}_{R}}$, then for each such coset $C=a_{C}+\mathfrak{N}_{R}$ of $\mathfrak{N}_{R}$ in $R, \sigma(C)=b_{C}+\mathfrak{N}_{R}$ for some representative $b_{C} \in \mathcal{R}$; in particular, there is a permutation $\sigma_{C} \in S_{n}$ such that for each $i=1, \ldots, n, \sigma\left(a_{C}+x_{i}\right)=b_{C}+x_{\sigma_{C}(i)}$. We now define $f(\sigma)=\left(\bar{\sigma}, \prod_{C \in R / \mathfrak{N}_{R}} \sigma_{C}\right)$; it is immediate that $f$ is a homomorphism and that Ker $f=1$, so $f$ is injective.

Now suppose we are given $\psi=\left(\tau, \prod_{C \in R / \mathfrak{N}_{R}} \phi_{C}\right) \in \operatorname{Aut}\left(G_{R_{\text {red }}}\right) \times \prod_{C \in R / \mathfrak{N}_{R}} S_{n}$. By construction, each element of $R$ may be written uniquely as $a_{C}+x_{j}$ for some $C \in R / \mathfrak{N}_{R}$ and $1 \leqslant j \leqslant n$. Define $b_{C}$ to be the (unique) element of $\mathcal{R}$ satisfying $\tau\left(a_{C}+\mathfrak{N}_{R}\right)=b_{C}+\mathfrak{N}_{R}$. Now define $\sigma \in \operatorname{Aut}\left(G_{R}\right)$ by $\sigma\left(a_{C}+x_{j}\right)=b_{C}+\phi_{C}\left(x_{j}\right)$. Then $f(\sigma)=\psi$ and so $f$ is surjective.

For rings $S_{1}, \ldots, S_{m}$, we define the number of leading zeros of an element $s=\left(s_{1}, \ldots\right.$, $\left.s_{m}\right) \in S_{1} \times \ldots \times S_{m}$ to be $\max \left\{\ell \geqslant 0: s_{1}=\ldots=s_{\ell}=0\right\}$.

We now turn to the case of reduced rings.

Theorem 4.2. Let $s \geqslant 1$, and suppose $r_{1}, \ldots, r_{s}$ are prime powers such that $2 \leqslant r_{1}<$ $\ldots<r_{s}$. For each $i=1, \ldots, s$, let $n_{i} \geqslant 1$ be an integer, and consider the ring $R=$ $\prod_{i=1}^{s}\left(F_{i}\right)^{n_{i}}$, where $F_{i}$ denotes the field with $r_{i}$ elements. Then Aut $\left(G_{R}\right) \cong \prod_{i=1}^{s} S_{r_{i}} \times$ $\prod_{i=1}^{s} S_{n_{i}}$.

\section{Proof.}

The idea behind the proof is to identify certain "obvious" automorphisms of $G_{R}$ and then prove that any automorphism $\sigma$ coincides with one of these, using the property that for any two vertices $u, v \in G_{R}, N(\sigma(u), \sigma(v))=N(u, v)$.

Since $R$ is reduced, any (set) map $f: R \rightarrow R$ which fixes all but one of the local factors and permutes the elements of the remaining factor induces an automorphism of $G_{R}$. Similarly, a map $f: R \rightarrow R$ which is the identity on $\left(F_{i}\right)^{n_{i}}$ for $i \neq i_{0}$ and permutes the $n_{i_{0}}$ factors of the form $F_{i_{0}}$ induces an automorphism of $G_{R}$. Let $H \subseteq \operatorname{Aut}\left(G_{R}\right)$ be the subgroup generated by maps of either of these two types. It remains to check that in fact $H=\operatorname{Aut}\left(G_{R}\right)$. Observe that translations, i.e. automorphisms of the form $z \mapsto z+a$ for some fixed $a \in R$, are compositions of maps of the first type.

To this end, suppose $\sigma \in \operatorname{Aut}\left(G_{R}\right)$. Composing with a translation, we may assume without loss of generality that $\sigma(0)=0$. Our goal is prove that, after composition with maps in $H, \sigma(a)=a$ for all $a \in R$. We do this by downward induction on the number $\ell$ of leading zeros in the coordinate representation for $a$, the base case being $\ell=m$; that is, $a=0$.

Suppose by induction that $\sigma(a)=a$ for all $a$ with more than $\ell$ leading zeros, and suppose $b=\left(b_{1,1}, \ldots, b_{1, n_{1}}, \ldots, b_{s, 1}, \ldots, b_{s, n_{s}}\right) \in R$ has $\ell$ leading zeros. Suppose the leftmost 
nonzero coordinate in $b$ is the $(i, j)$ coordinate. Define $b^{\prime}$ to have the same coordinates as $b$ except for the $(i, j)$ coordinate, which is 0 . Observe that if $c \in R$ has at most $\ell$ leading zeros, then $\left|N\left(b, b^{\prime}\right)\right| \leqslant\left|N\left(c, b^{\prime}\right)\right|$ by Proposition 2.3, with equality if and only if $c$ and $b$ differ only in the $(i, k)$ coordinate for some $k, 1 \leqslant k \leqslant j$. By induction, $\sigma\left(b^{\prime}\right)=b^{\prime}$ and $\sigma(b)$ has at most $\ell$ leading zeros. Moreover, since $\sigma$ is an automorphism, $N\left(b, b^{\prime}\right)=N\left(\sigma(b), b^{\prime}\right)$, so by the inequality above, $\sigma(b)$ differs from $b$ only in the $(i, k)$ coordinate, where $1 \leqslant k \leqslant j$. By applying an automorphism in $H$ of the second type, we may assume that $k=j$, and after applying an automorphism of the first type, $\sigma(b)=b$. This completes the induction.

\section{Connectivity}

Proposition 5.1. Let $R$ be any finite ring, and let $\kappa\left(G_{R}\right)$ and $\kappa^{\prime}\left(G_{R}\right)$ denote (respectively) the vertex-connectivity and edge-connectivity of its unitary Cayley graph. Then $\kappa\left(G_{R}\right)=$ $\kappa^{\prime}\left(G_{R}\right)=\left|R^{*}\right|$.

\section{Proof.}

We argue following the reasoning in [9], Theorem 4. According to a theorem of Watkins [10], the vertex connectivity of a regular edge-transitive graph is equal to its degree of regularity. We show that $G_{R}$ is edge-transitive by observing that for any edge $\{u, v\}$ the automorphism $x \mapsto(v-u)^{-1}(x-u)$ maps $u$ to 0 and $v$ to 1 . Hence $\kappa\left(G_{R}\right)=\left|R^{*}\right|$. Since $\kappa\left(G_{R}\right) \leqslant \kappa^{\prime}\left(G_{R}\right) \leqslant\left|R^{*}\right|$ by $\left[11\right.$, Theorem 4.1.9], it follows that $\kappa\left(G_{R}\right)=\kappa^{\prime}\left(G_{R}\right)=\left|R^{*}\right|$.

\section{Clique Number, Chromatic Number, and Indepen- dence Number}

For a graph $G$, we denote by $\bar{G}$ its complement, $\omega(G)$ its clique number, $\alpha(G)$ its independence number, and $\chi(G)$ its chromatic number.

Proposition 6.1. Let $R$ be a finite ring. Then $\omega\left(G_{R}\right)=\chi\left(G_{R}\right)=f_{1}$ and $\omega\left(\overline{G_{R}}\right)=$ $\chi\left(\overline{G_{R}}\right)=\alpha\left(G_{R}\right)=\frac{|R|}{f_{1}}$.

\section{Proof.}

Choose elements $r_{i j} \in R_{i}, i=1, \ldots, t, j=1, \ldots, f_{1}$ such that for each $i=1, \ldots, t$ and $j \neq j^{\prime}, \pi_{i}\left(r_{i j}\right) \neq \pi_{i}\left(r_{i j^{\prime}}\right)$. Then, setting $a_{j}=\left(r_{1 j}, \ldots, r_{t j}\right)$ for each $j=1, \ldots, f_{1}$, it is easily seen that $C=\left\{a_{1}, \ldots, a_{f_{1}}\right\}$ is a clique and $\omega\left(G_{R}\right) \geqslant f_{1}$. Now consider the ideal $I=\mathfrak{m}_{1} \times R_{2} \ldots \times R_{t} \subseteq R$. There are precisely $f_{1}$ cosets of $I$ in $R$, each of which corresponds to an independent subset of $G_{R}$. By assigning each coset a distinct color and coloring all vertices within that coset the same color, we have constructed a proper coloring of $G_{R}$. Hence, $\chi\left(G_{R}\right) \leqslant f_{1}$. Since $f_{1} \leqslant \omega\left(G_{R}\right) \leqslant \chi\left(G_{R}\right) \leqslant f_{1}$, we have $\omega\left(G_{R}\right)=\chi\left(G_{R}\right)=f_{1}$. 
Since the ideal $I$ constructed above corresponds to an independent set in $G_{R}$, we have $\alpha\left(G_{R}\right)=\omega\left(\overline{G_{R}}\right) \geqslant|I|=|R| / f_{1}$. We now construct a coloring of $\overline{G_{R}}$ by elements of $I$ as follows: given $b=\left(b_{1}, \ldots, b_{n}\right) \in R$, fix a clique $C$ in $G_{R}$ as above and let $c_{b}$ be the unique element of $C$ such that $b-c_{b} \in I$; define a vertex coloring $f: R \rightarrow I$ by $f(b)=b-c_{b}$. Then $f(b)=f(d)$ implies that $b-d=c_{d}-c_{b}$. If $c_{d}=c_{b}$, then $b=d$; so assume $c_{d} \neq c_{b}$. Then by construction, $c_{d}-c_{b} \in R^{*}$, so $b-d \in R^{*}$, and hence $b$ is not adjacent to $d$ in $\overline{G_{R}}$. Thus $f$ is a proper coloring, showing that $\chi\left(\overline{G_{R}}\right) \leqslant|I|=|R| / f_{1}$, as desired.

Corollary 6.2. Let $R$ be a finite ring. Then $G_{R}$ is $f_{1}$-partite.

\section{$7 \quad$ Edge Chromatic Number}

We next derive a result concerning the edge chromatic number $\chi^{\prime}\left(G_{R}\right)$.

Theorem 7.1. Let $R$ be a finite ring. Then

$$
\chi^{\prime}\left(G_{R}\right)= \begin{cases}\left|R^{*}\right|+1 & \text { if }|R| \text { is odd } \\ \left|R^{*}\right| & \text { if }|R| \text { is even. }\end{cases}
$$

\section{Proof.}

Since $G_{R}$ is $\left|R^{*}\right|$-regular, $\chi^{\prime}\left(G_{R}\right) \geqslant\left|R^{*}\right|$, and by Vizing's Theorem, $\chi^{\prime}\left(G_{R}\right) \leqslant\left|R^{*}\right|+1$. Suppose $|R|$ is odd, so $G_{R}$ has no 1-factor. Then in any proper edge-coloring of $G_{R}$, each color class must miss some vertex $x$. Hence there are $\left|R^{*}\right|$ colors used on edges incident at $x$, plus the color of that class used elsewhere; hence, $\chi^{\prime}\left(G_{R}\right)=\left|R^{*}\right|+1$.

Now suppose $|R|$ is even. By Proposition 2.1, at least one of the local rings in the decomposition $R \cong R_{1} \times \ldots \times R_{t}$ has even cardinality. In particular, this means that for any unit $u=\left(u_{1}, \ldots, u_{t}\right) \in R^{*},|u|=\operatorname{lcm}\left(\left|u_{1}\right|, \ldots,\left|u_{t}\right|\right)$ is even, where by $|u|\left(\right.$ or $\left.\left|u_{i}\right|\right)$ we mean the order of $u$ as an element of the additive abelian group $R$ (respectively, $R_{i}$ ).

Let $V=\left\{v \in R^{*}:|v|=2\right\}$ and $E_{V}=\{\{r, r+v\}: v \in V\} \subseteq E\left(G_{R}\right)$. We observe that there are exactly $|V|$ edges of $E_{V}$ incident at every vertex of $G_{R}$. Now construct a proper coloring of $E(G)$ as follows: fix a bijection $h: V \rightarrow\{1, \ldots,|V|\}$ and, for each $v \in V$ and $r \in R$, color the edge $\{r, r+v\}$ with color $h(v)$. Now let $U^{\prime}=R^{*}-V$; note that for each $u \in U^{\prime}, u \neq-u$. Choose $U=\left\{u_{1}, \ldots, u_{m}\right\} \subseteq U^{\prime}$ such that for all $u \in U^{\prime}$,

exactly one of $u,-u$ is in $U$; thus, $|U|=\frac{\left|R^{*}\right|-|V|}{2}$. Now for each $u_{j}, j=1, \ldots, m$, let $a_{1}+<u_{j}>, \ldots, a_{s}+<u_{j}>$ be the (distinct) cosets of $<u_{j}>$ in $R$. For each $k=0, \ldots,\left|u_{j}\right|-1$, color the edge $\left\{a_{i}+k u_{j}, a_{i}+(k+1) u_{j}\right\}$ with color $|V|+2 j-1$ if $k$ is odd or color $|V|+2 j$ if $k$ is even. It is easy to check that this procedure defines a proper edge-coloring of $G$ with $2|U|+|V|=\left|R^{*}\right|$ colors.

\section{Planarity}

The following is immediate from definitions: 
Lemma 8.1. Let $G$ be a bipartite graph. Then $G \wedge K_{2} \cong 2 G$. In particular, $G$ is planar if and only if $G \wedge K_{2}$ is planar.

Our result on planarity is:

Theorem 8.2. Let $R$ be a finite ring. Then $G_{R}$ is planar if and only if $R$ is one of the following rings: $(\mathbb{Z} / 2 \mathbb{Z})^{s}, \mathbb{Z} / 3 \mathbb{Z} \times(\mathbb{Z} / 2 \mathbb{Z})^{s}, \mathbb{Z} / 4 \mathbb{Z} \times(\mathbb{Z} / 2 \mathbb{Z})^{s}$, or $\mathbb{F}_{4} \times(\mathbb{Z} / 2 \mathbb{Z})^{s}$. (Here $\mathbb{F}_{4}$ is the field with four elements and $s \geqslant 0$ may assume any integer value.)

\section{Proof.}

Clearly, $\mathbb{Z} / 2 \mathbb{Z}$ and $\mathbb{Z} / 3 \mathbb{Z}$ are the only rings with fewer than 4 elements, so henceforth let $R$ be a finite ring such that $G_{R}$ is planar and $|R| \geqslant 4$.

If $f_{1}=2$, then $R \cong R_{1} \times \ldots \times R_{t}$ is bipartite by Corollary 6.2 and as such is trianglefree. By a well-known result (see for example [11, Theorem 6.1.23]), planarity of $R$ forces

$|E(R)| \leqslant 2|R|-4$; that is, $\left|R^{*}\right| \leqslant 4-\frac{8}{|R|}$ or $\left|R^{*}\right| \leqslant 3$. Now if $S$ is a local ring, $\left|S^{*}\right| \geqslant \frac{|S|}{2} ;$ hence, $\left|S^{*}\right|=1$ if and only if $S \cong \mathbb{Z} / 2 \mathbb{Z}$. Moreover, $R^{*}=R_{1}^{*} \times \ldots \times R_{t}^{*}$, so the condition $\left|R^{*}\right| \leqslant 3$ forces $R \cong S \times\left(\mathbb{Z}_{2}\right)^{s}$ for some $s \geqslant 0$ and some local ring $S$ with $\left|S^{*}\right| \leqslant 3$. Since $|S| \leqslant 6$ and $|S|$ must be a prime power, the only possibilities are $S=\mathbb{Z} / 2 \mathbb{Z}, \mathbb{Z} / 3 \mathbb{Z}, \mathbb{Z} / 4 \mathbb{Z}$ (with any $s \geqslant 0$ ), or $S=\mathbb{F}_{4}$ (with any $s \geqslant 1$ ). It is easy to check by hand that for each of these choices of $S$, both $G_{S}$ and $G_{S \times \mathbb{Z} / 2 \mathbb{Z}}$ are planar. Since the graph $G_{S \times \mathbb{Z} / 2 \mathbb{Z}}$ is guaranteed to be bipartite by Corollary 6.2, planarity of $S \times\left(\mathbb{Z}_{2}\right)^{s}$ follows from Lemma 8.1 by induction.

Now suppose $f_{1} \geqslant 3$. Then (cf. [11, Theorem 6.1.23]) planarity of $R \cong R_{1} \times \ldots \times R_{t}$ forces $|E(R)| \leqslant 3|R|-6$, which implies $\left|R^{*}\right| \leqslant 5$. However, this time each of the local factors $R_{i}$ satisfies $\left|R_{i}^{*}\right| \geqslant \frac{2}{3}\left|R_{i}\right|$; in particular, if $\left|R_{i}^{*}\right|=2$, then $\left|R_{i}\right|=3$ and hence $R_{i} \cong \mathbb{Z} / 3 \mathbb{Z}$, which is impossible since $f_{1} \geqslant 3$. If $\left|R^{*}\right|=3$, then $R \cong \mathbb{F}_{4}$, and if $\left|R^{*}\right|=4$, then $R \cong \mathbb{Z} / 5 \mathbb{Z}$. Clearly $G_{\mathbb{F}_{4}} \cong K_{4}$ is planar but $G_{\mathbb{Z} / 5 \mathbb{Z}} \cong K_{5}$ is not.

\section{$9 \quad$ Perfectness}

Let $R$ be an Artinian ring. In this section, we classify which of the graphs $G_{R}$ are perfect. As before, fix a decomposition $R \cong R_{1} \times \ldots \times R_{t}$ as a product of local rings. We note that our proof, while following the outline of the analogous result in Section 3 of [9], differs somewhat in that it avoids use of the Fuchs-Sinz result [8] on longest induced cycles.

If $t=1$ then by Proposition $2.2 G_{R}$ is complete multipartite and hence is perfect. If $f_{1}=2$ then by Corollary $6.2 G_{R}$ is bipartite and hence perfect. We assume henceforth that $f_{1} \geqslant 3$. Our main tool is the Strong Perfect Graph Theorem.

Theorem 9.1. [3] A graph $G$ is perfect if and only if neither $G$ nor $\bar{G}$ contains an induced odd cycle.

Lemma 9.2. Suppose $t \geqslant 3$. Then $G_{R}$ is not perfect. 


\section{Proof.}

For each $i=1, \ldots, t$, fix elements $a_{i}^{(0)}, a_{i}^{(1)}, a_{i}^{(2)}$ such that the values of $\pi_{i}\left(a_{i}^{(j)}\right), j=$ $0,1,2$, are mutually distinct. For convenience, we assume $a_{i}^{0}=0, a_{i}^{(1)}=1$ for all $i$ and write $c_{i}=a_{i}^{(2)}$. Then given a triple $t=\left(a_{1}^{\left(x_{1}\right)}, a_{2}^{\left(x_{2}\right)}, a_{3}^{\left(x_{3}\right)}\right) \in R_{1} \times R_{2} \times R_{3}$, where $0 \leqslant x_{j} \leqslant 2$ for $j=1,2,3$, define its extension $\operatorname{ext}(t)=\left(w_{1}, \ldots, w_{t}\right) \in R$ by $w_{i}=a_{i}^{\left(x_{j}\right)}$, where $0 \leqslant j \leqslant 2$ is the unique integer such that $i \equiv j(\bmod 3)$. Then the vertices $\operatorname{ext}(0,0,0), \operatorname{ext}(1,1,1), \operatorname{ext}\left(0, c_{2}, c_{3}\right), \operatorname{ext}(1,1,0), \operatorname{ext}\left(c_{1}, c_{2}, 1\right)$ induce a 5 -cycle in $G_{R}$.

Hence we are reduced to the case that $R \cong R_{1} \times R_{2}$ where $R_{1}, R_{2}$ are local.

Lemma 9.3. If $R$ is of the above form, then $\overline{G_{R}}$ does not contain any induced odd cycle of length $\geqslant 5$.

\section{Proof.}

For contradiction, suppose that $\overline{G_{R}}$ has an induced cycle of length $2 m+1$ for some $m \geqslant 2$, and that the order of consecutive vertices around the cycle is $a_{1}, \ldots, a_{2 m+1}, a_{1}$. For each $i$, let $a_{i}=\left(a_{i, 1}, a_{i, 2}\right)$. Then, since $a_{i}$ is adjacent to $a_{i+1}$ (taken modulo $\left.2 \mathrm{~m}+1\right)$ in $\overline{G_{R}}$, at least one of $\pi_{1}\left(a_{i, 1}\right)=\pi_{1}\left(a_{i+1,1}\right)$ or $\pi_{2}\left(a_{i, 2}\right)=\pi_{2}\left(a_{i+1,2}\right)$ must hold. For convenience, call the edge $\left\{a_{i}, a_{i+1}\right\}$ red if the first statement holds or blue otherwise. Because $a_{i-1}$ is also adjacent to $a_{i}$ but not to $a_{i+1},\left\{a_{i-1}, a_{i}\right\}$ cannot be the same color as $\left\{a_{i}, a_{i+1}\right\}$. Hence, consecutive edges around the cycle alternate between red and blue; however, this leads to a contradiction because the cycle has odd length.

Lemma 9.4. If $R$ is of the above form, then $G_{R}$ does not contain any induced odd cycle of length $\geqslant 5$.

\section{Proof.}

Suppose first that $m \geqslant 3$ and the subgraph induced by some vertices $a_{i}=\left(a_{i, 1}, a_{i, 2}\right)$, $i=1, \ldots, 2 m+1$ is a cycle. As above, assume that the order of consecutive vertices around the cycle is given by $a_{1}, a_{2}, \ldots, a_{2 m+1}, a_{1}$. After applying an appropriate automorphism of $G_{R}$, we may assume $a_{1}=(0,0)$ and $a_{2}=(1,1)$. Since $a_{3}$ is not adjacent to $a_{1}$, at least one of $\pi_{1}\left(a_{3,1}\right), \pi_{2}\left(a_{3,2}\right)$ is 0 . However, since $a_{5}$ is adjacent to neither $a_{1}$ nor $a_{3}$, we may assume without loss of generality that $\pi_{1}\left(a_{3,1}\right)=\pi_{1}\left(a_{5,1}\right)=0$. On the other hand, $a_{5}$ is not adjacent to $a_{2}$, so $\pi_{2}\left(a_{5,2}\right)=1$. Moreover, $a_{4}$ is adjacent to $a_{5}$, but not to $a_{1}$ or $a_{2}$, so $\pi_{1}\left(a_{4,1}\right)=1$ and $\pi_{2}\left(a_{4,2}\right)=0$. Also, since $a_{3}$ is adjacent to $a_{4}, \pi_{2}\left(a_{3,2}\right) \neq 0$. Finally, $a_{6}$ is not adjacent to $a_{1}$; if $\pi_{1}\left(a_{6,1}\right)=0$, this contradicts its being adjacent to $a_{5}$. Hence $\pi_{1}\left(a_{6,3}\right) \neq 0$ and $\pi_{2}\left(a_{6,2}\right)=0$, but since $a_{6}$ is not adjacent to $a_{2}$, it must be the case that $\pi_{1}\left(a_{6,2}\right)=1$; this contradicts $a_{6}$ not being adjacent to $a_{3}$.

The assertion for cycles of length 5 follows from Lemma 9.3 and the fact that the complement of a 5-cycle is another 5-cycle.

The results above now prove:

Theorem 9.5. Let $R$ be an Artinian ring. Then $G_{R}$ is perfect if and only if $f_{1}=2, R$ is local, or $R$ is a product of two local rings. 


\section{Eigenvalues}

In this section, we show how to compute the eigenvalues of $G_{R}$ using elementary methods. The derivation we give is much shorter and simpler than the proof in [9] (which involves Ramanujan sums) and hinges on the property that $G_{R}$ is a conjunction of complete multipartite graphs.

Let $R$ be a finite ring with local factors $R_{1}, \ldots, R_{t}$. As is standard, if $A$ is an $n \times n$ matrix with eigenvalues $\lambda_{1}, \ldots, \lambda_{n}$ of respective multiplicities $m_{1}, \ldots, m_{n}$, we use the notation Spec $A=\left(\begin{array}{ccc}\lambda_{1} & \ldots & \lambda_{n} \\ m_{1} & \ldots & m_{n}\end{array}\right)$ to describe the spectrum of $A$.

Lemma 10.1. Let $G$ and $H$ be graphs. Suppose that $\lambda_{1}, \ldots, \lambda_{n}$ are the eigenvalues of $G$ and $\mu_{1}, \ldots, \mu_{n}$ are the eigenvalues of $H$ (repetition is possible). Then the eigenvalues of $G \wedge H$ are $\lambda_{i} \mu_{j}, 1 \leqslant i \leqslant n, 1 \leqslant j \leqslant n$.

\section{Proof.}

The result follows immediately from the well-known facts that $A(G \wedge H)$ is the tensor product of the matrices $A(G)$ and $A(H)$, and that the eigenvalues of a tensor product of matrices may be found by taking products of the eigenvalues of the factors.

The fundamental results are contained in the following routine calculation:

\section{Proposition 10.2.}

- Let $F$ be a field with $n$ elements. Then $\operatorname{Spec}\left(G_{F}\right)=\left(\begin{array}{cc}n-1 & -1 \\ 1 & n-1\end{array}\right)$.

- Let $S$ be a finite local ring which is not a field, having (nonzero) maximal ideal $\mathfrak{m}$ of size $m$. Let $f=|S| / m$. Then Spec $\left(G_{S}\right)=\left(\begin{array}{cc}-m & 0 \\ f & f(m-1)\end{array}\right)$.

\section{Proof.}

If $F$ is a field with $n$ elements, $G_{F} \cong K_{n}$. Its adjacency matrix is $A\left(G_{F}\right)=J_{n}-I_{n}$, where $J_{n}$ is the matrix of all 1 s and $I_{n}$ is the identity matrix. Hence, the eigenvalues of $A\left(G_{F}\right)$ are each 1 less than those of $J_{n}$. To determine the latter, $J_{n}$ is clearly seen to have rank 1 , so 0 is an eigenvalue of multiplicity $n-1$. Moreover, the vector $[1, \ldots, 1]^{T}$ is clearly seen to be an eigenvector of $J_{n}$ with associated eigenvalue $n$, which must necessarily be of multiplicity 1 .

If $S$ is a local ring with maximal ideal $\mathfrak{m} \neq 0$, then $G_{S}$ is a balanced complete multipartite graph with $f=|S / \mathfrak{m}|$ partite sets, each of size $m=|\mathfrak{m}|$. In view of the regularity of $G_{S}$, it is well-known (cf. [11, Theorem 8.6.25]) that if $\lambda_{1}, \ldots, \lambda_{n}$ are eigenvalues for $A\left(G_{S}\right)$, then $-1-\lambda_{1}, \ldots,-1-\lambda_{n}$ are eigenvalues for $A\left(\overline{G_{S}}\right)$. However, $\overline{G_{S}}$ is a disjoint union of $f$ cliques, each of size $m$; hence $\operatorname{Spec}\left(\overline{G_{S}}\right)=\left(\begin{array}{cc}m-1 & -1 \\ f & f(m-1)\end{array}\right)$ and so $\operatorname{Spec}\left(G_{S}\right)=\left(\begin{array}{cc}-m & 0 \\ f & f(m-1)\end{array}\right)$. 
It is easily seen that these calculations, together with Lemma 10.1, may be used to compute the eigenvalues of $G_{R}$ for any ring $R$. Since the eigenvalues of $G_{F}$ are all nonzero when $F$ is a field, the formula for the spectrum of $G_{R}$ becomes quite complicated when many of the local factors of $R$ are fields. However, if none of the local factors of $R$ are fields, the formula takes on a rather appealing form:

Corollary 10.3. Let $R$ be a finite ring and suppose $R$ has $t$ local factors, none of which are fields. Then Spec $\left(G_{R}\right)=\left(\begin{array}{cc}(-1)^{t}\left|\mathfrak{N}_{R}\right| & 0 \\ \left|R_{\text {red }}\right| & |R|-\left|R_{\text {red }}\right|\end{array}\right)$.

\section{Proof.}

Suppose the local factors of $R$ are $R_{i}, i=1, \ldots, t$, each $R_{i}$ having maximal ideal of size $m_{i}>1$ and residue field of size $f_{i}$. Then the previous calculation and Lemma 10.1 together imply

$$
\operatorname{Spec}\left(G_{R}\right)=\left(\begin{array}{cc}
(-1)^{t} \prod_{i=1}^{t} m_{i} & 0 \\
\prod_{i=1}^{t} f_{i} & |R|-\prod_{i=1}^{t} f_{i}
\end{array}\right)=\left(\begin{array}{cc}
(-1)^{t}\left|\mathfrak{N}_{R}\right| & 0 \\
\left|R_{\text {red }}\right| & |R|-\left|R_{\text {red }}\right|
\end{array}\right) .
$$

\section{References}

[1] M. Bernstein. Representations of graphs and rings. SUMSRI Journal, 2008.

[2] P. Berrizbeitia and R. E. Giudici. On cycles in the sequence of unitary Cayley graphs. Discrete Math. 282 (2004), 1-3.

[3] M. Chudnovsky, N. Robertson, P. Seymour, and R. Thomas. The Strong Perfect Graph Theorem. Ann. Math. 164 (2002), 51-229.

[3] I. Dejter and R. E. Giudici. On unitary Cayley graphs. J. Combin. Math. Combin. Comput. 18 (1995), 121-124.

[4] D. S. Dummit and R. M. Foote. Abstract Algebra. Third Edition, Wiley and Sons.

[5] P. Erdös and A. B. Evans. Representations of graphs and orthogonal Latin square graphs. J. Graph Theory 13 (1989), no. 5, 593-595.

[6] A. B. Evans, G. Fricke, C. Maneri, T. McKee, M. Perkel. Representations of Graphs Modulo n. Journal of Graph Theory 18, no. 8 (1994), 801-815.

[7] E. Fuchs. Longest induced cycles in circulant graphs. Electronic Journal of Combinatorics 14 (2005), no. 1, Research Paper 52.

[8] E. Fuchs and J. Sinz. Longest induced cycles in Cayley graphs. ArXiv: math/0410308v2 (2004).

[9] W. Klotz and T. Sander. Some Properties of Unitary Cayley Graphs. Electronic Journal of Combinatorics 14 (2007), no. 1, Research Paper 45.

[10] M. E. Watkins. Connectivity of transitive graphs. J. Combin. Theory 8 (1970), 23-29.

[11] D. B. West. Graph Theory, Second Edition. Prentice-Hall, 2000. 